\title{
A Current-Mode Interface Circuit for a Piezoresistive Pressure Sensor
}

Josep Samitier, Associate Member, IEEE, Manel Puig-Vidal, Member, IEEE, Sebastian A. Bota, Carles Rubio, Stilianos K. Siskos, Member, IEEE, Theordore Laopoulos, Senior Member, IEEE

\begin{abstract}
An interfacing circuit for piezoresistive pressure sensors based on CMOS current conveyors is presented. The main advantages of the proposed interfacing circuit include the use of a single piezoresistor, the capability of offset compensation, and a versatile current-mode configuration, with current output and current or voltage input. Experimental tests confirm linear relation of output voltage versus piezoresistance variation.
\end{abstract}

Index Terms - CMOS analog integrated circuit, current conveyor, current mode, interface circuit, pressure sensor.

\section{INTRODUCTION}

D URING the last few years, integrated pressure sensors on silicon substrate are of growing interest because of their compatibility with CMOS technology. The main advantages of these systems are their improved sensitivity, small size, and cost effectiveness. Silicon pressure sensors that utilize the piezoresistive effect are usually based in a fourelement Wheatstone bridge configuration, formed by two $R_{p}^{+}$ elements (their resistance increases with pressure) and two $R_{p}^{-}$elements (their resistance decreases with pressure). The output voltage of such a transducer is sensed by an interface circuit usually based on operational amplifiers [1]. In these four-element pressure sensors, accurate resistance matching between $R_{p}^{+}$and $R_{p}^{-}$and equal variation of absolute resistance with pressure are two essential characteristics for proper operation to reduce the offset and increase the sensitivity of the sensor. These constraints complicate the technological process to implement $R_{p}^{+}$and $R_{p}^{-}$elements.

The use of a single piezoresistance element eliminates the need to match closely the four resistors that form the Wheatstone bridge design [2]. A novel cost-effective currentmode interfacing circuit designed to operate with input from a single piezoresistance element is presented in this paper. The proposed circuit, based on second-generation current conveyors (CCII + ) [3], also offers current output which can be very useful in a number of applications in which relatively long wires are used to connect the sensor to the monitoring system. Such is the case in biomedical systems where the proposed configuration is to be used. The pressure sensor integrated on the chip with the proposed interfacing circuit forms a sensing

Manuscript received September 29, 1995; revised November 30, 1998. This work was supported in part by the Spanish CICYT through Project TAP941047 and Project TIC95-17 08-CE.

J. Samitier, M. Puig-Vidal, S. A. Bota, and C. Rubio are with Laboratori de Sistemes Electronics d'Intrumentacio i Comunicacions (SEI\&C), Departamen Electronica, Facultat de Fisica, Universitat Barcelona, 08028 Barcelona, Spain.

S. K. Siskos and T. Laopoulos are with the Electronics Laboratory, Physics Department, Aristotle University Thessaloniki, 54006 Thessaloniki, Greece.

Publisher Item Identifier S 0018-9456(98)09794-0.

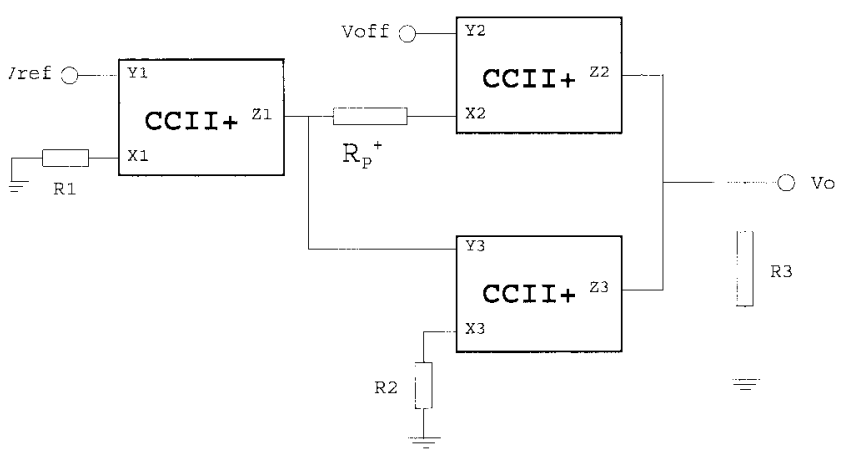

Fig. 1. Block diagram of the interface circuit. Piezoresistance is denoted by $R_{p}^{+}=R_{p 0}^{+}(1+x)$.

element. Such an element may be directly connected to the external patient-monitoring system with increased signal-noise immunity and transmission efficiency.

\section{CirCUIT DESCRIPTION}

The second-generation current conveyor CCII + is a known current-mode three-port analog building block with the following characteristics: $V_{x}=V_{y}, I_{x}=I_{z}$, and $I_{y}=0$ [3]. The proposed interface involves three such CCII + cells, three conventional resistors $R_{1}, R_{2}, R_{3}$ (the last one at the monitoring instrument side), and a single piezoresistor, denoted as $R_{p}^{+}=R_{p 0}^{+}(1+x)$, where $R_{p 0}^{+}$is the resistance at reference pressure and $x=\Delta R / R_{p 0}^{+}$is the relative change proportional to pressure variation. The resulting circuit schematic diagram is depicted in Fig. 1.

A current source configuration is obtained via the first CCII + . This current value $I_{Z 1}$ is equal to $I_{x 1}$ and therefore determined by reference voltage $V_{\text {ref }}$ and resistance $R_{1}$. The applied pressure produces an absolute change $\Delta R$ in the piezoresistor $R_{p}^{+}$. The small change in piezoresistor value affects the voltage at node $Z 1$

$$
\begin{aligned}
V_{z 1}-V_{\mathrm{off}} & =I_{z 1} R_{p}^{+} \\
V_{z 1} & =I_{z 1} R_{p 0}^{+}(1+x)+V_{\mathrm{off}} \\
& =\frac{V_{\text {ref }}}{R_{1}} R_{p 0}^{+}(1+x)+V_{\text {off }} .
\end{aligned}
$$

According to the CCII + operation, the output current of the second CCII + also follows the input current $\left(I_{z 2}=-I_{x 1}\right)$ since $Y 3$ is a high impedance node and does not affect the value of the current through the piezoresistor. On the other hand, the output current of the third CCII + will be controlled by $V_{z 1}$ and $R_{2}\left(I_{Z 3}=V_{Z 1} / R_{2}\right)$. The two currents $I_{z 2}$ and $I_{z 3}$ are then added to obtain the output current $I_{O}$ and, 
consequently, the output voltage will be

$$
V_{o}=\left(I z_{3}-I z_{2}\right) R_{3}
$$

Therefore, a linear dependence between the output current $I_{o}$ and the relative piezoresistor variation $x$ is obtained and can be expressed as follows:

$$
\begin{aligned}
I o & =I z_{3}=I z_{2}=\frac{V_{z 1}}{R_{2}}-\frac{V_{\text {ref }}}{R_{1}} \\
& =V_{\text {ref }} \frac{R_{p 0}^{+}}{R_{2} R_{1}} x+\frac{V_{\text {ref }}}{R_{1}}\left(\frac{R_{p 0}^{+}}{R_{2}}-1\right)+\frac{V_{\text {of }}}{R_{2}} .
\end{aligned}
$$

In order to eliminate the constant output offset value formed by the last two terms, either $R_{2}$ has to be matched with the initial piezoresistor value $R_{p 0}^{+}$and $V_{\text {off }}=0$, or it could be also compensated by an appropriate external voltage applied to the input $V_{\text {off }}$. Then, after the output offset value correction, the final output value is

$$
I_{0}=V_{\text {ref }} \frac{R_{p 0}^{+}}{R_{1} R_{2}} x=k x .
$$

A current output proportional to the pressure variation is obtained while the resistors' ratio $R_{3} / R_{1}$ may be used to control the output voltage gain of the compensated circuit. It should also be noted that both reference and offset inputs are high impedance nodes and therefore are practically not affected by the length of connection wires, although $V_{\text {ref }}$ may be also integrated with this circuit. Moreover, the proposed configuration can be used in a true current-mode operation with current reference input at node $x_{1}$ and current output, and this is difficult to obtain with operational amplifiers.

\section{EXPERIMENTAL RESULTS}

The proposed circuit of Fig. 1 has been implemented using CMOS current conveyors cells previously fabricated in an 1.2$\mu \mathrm{m}$ CMOS process [4]. Experimental results were obtained using a typical piezoresistance (with $R_{p 0}^{+}=2.391 \mathrm{k} \Omega$ ) with a $10 \%$ variation due to pressure changes [5]. The experimental fixed resistance values were set to $R_{1}=2.395 \mathrm{k} \Omega, R_{2}=$ $2.393 \mathrm{k} \Omega, R_{3}=23.926 \mathrm{k} \Omega$ (in order to set the gain factor to 10). Reference voltage is fixed at $V_{\text {ref }}=0.209 \mathrm{~V}$ and an offset compensation is applied at $V_{\text {off }}=-2.24 \mathrm{mV}$. A typical experimental measurement set for the proposed circuit and its linear regression are shown in Fig. 2. A linear dependence of output $V_{o}$ versus resistance variation $x$ is obtained. The nonlinearity measured using a best fit straight-line for absolute minimum squared error is less than $1 \%$ (full scale output). Furthermore, the whole interfacing circuit provides a relatively good frequency response up to $250 \mathrm{kHz}$.

\section{CONCLUSION}

A novel configuration for a pressure sensor interfacing circuit based on CMOS current conveyors has been designed and tested. This circuit uses a single piezoresistive element, thus avoiding the problems of the traditional four-element pressure sensors related to the matching between $R_{p}^{+}$and $R_{p}^{-}$ piezoresistances. Monolithic implementation of the pressure sensor plus the interface circuit in a single chip is currently

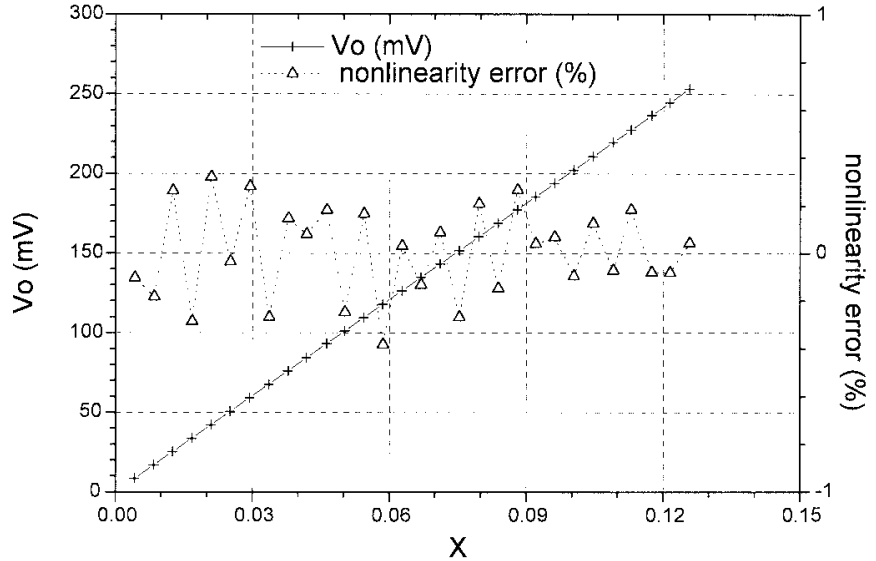

Fig. 2. Circuit output voltage and linearity error versus piezoresistor variation rate $x=\Delta R / R_{p 0}^{+}$.

in progress. This interface circuit is well suited for surgical cardiovascular applications for accurate intravascular pressure measurements. The pressure sensor plus this electronic interface circuit fulfill specific characteristics: minimum number of wires, minimum power dissipation, high noise immunity of the signal transmission, and accurate pressure-voltage conversion.

\section{ACKNOWLEDGMENT}

The authors would like to thank the anonymous reviewers for their helpful and constructive comments.

\section{REFERENCES}

[1] J. M. Borky and K. D. Wise, "Integrated signal conditioning for silicon pressure sensors," IEEE Trans. Electron Devices, vol. ED-26, pp. 1906-1910, Dec. 1979.

[2] R. Frank, "Pressure sensors merge micromachining and microelectronics," Sens. Actuators A, pp. 93-103, Jan. 1991.

[3] A. S. Sedra and G. W. Roberts, "Current conveyor theory and practice" in Analogue IC Design: The Current Mode Approach, C. Toumazou, F. J. Lidgey, and D. G. Haigh, Eds. London, U.K.: Peter Peregrinus, Ltd., 1990, ch. 3, pp. 93-126.

[4] T. Laopoulos, S. Siskos, M. Bafleur, and P. Givelin, "CMOS current conveyor," Electron. Lett., vol. 28, no. 24, pp. 2261-2262, Nov. 1992.

[5] S. Marco, J. Samitier, O. Ruíz, J. R. Morante, and J. Esteve, "High performance piezoresistive pressure transducer using very thin structured membranes" Meas. Sci. Technol., pp. 1195-1203, 1996.

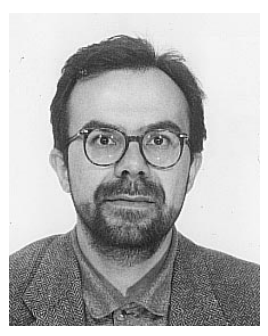

Josep Samitier (M'95-A'95) studied physics at the University of Barcelona, Barcelona, Spain, from 1977 to 1982 and received the Ph.D. degree from the same university in 1986.

He has been a Full Professor of the Electronic Department at the University of Barcelona, Barcelona, Spain, since February 1995, where he coordinates the research projects on Electronic Instrumentation He was Research Fellow during 1983 and 1984 in the Applied Physics Department of the University of Barcelona in the field of GaAs MESFET Devices and electrooptical characterization of III-V semiconductors. From February 1984 to June 1985, he was a Visiting Research Fellow at the Laboratoire D'Electronique Philips, LEP, Paris, France. In 1988, he was appointed an Assistant Professor of Electronics at the same university, working in the development of microsystems devices. Current research and development projects concern the design, test, and signal conditioning of microsystems, the design of interface circuits for sensors and microsystems, and the development of smart sensor and actuator networks based on field buses. Since 1995, he has been the Deputy Head of Electronic Engineering at the University of Barcelona. 


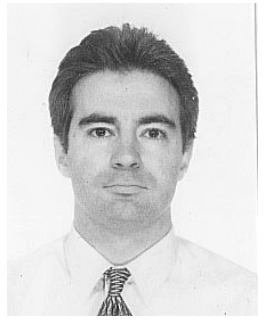

Manel Puig-Vidal (M'94) was born in Igualada, Barcelona, Spain, in October 1965. He received the M.S. degree in physics from the University of Barcelona, Barcelona, Spain, in 1988 and the Ph.D. degree from the University Paul Sabatier, Toulouse, France, in 1993.

From 1989 to 1993, he was Research Fellow in the Laboratoire d'Automatique et d'Analyze des Systèmes (LAAS), Toulouse, France, working in Latch-up free Smart-Power technology for automotive applications. In 1993, he was appointed an Assistant Professor of Electronics at the University of Barcelona, working in the field of power electronics. Since 1995, he has been Professor Titular at the University of Barcelona, teaching Power Electronics and Control Systems in Electronic Engineering. Current research and development projects concern high-voltage smart-power integrated circuits for microsystems.

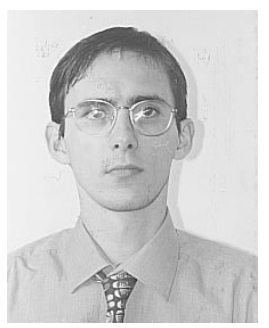

Sebastian A. Bota was born in Pollença, Spain, in 1964. He received the degree in physics in 1987 and Ph.D. degree in microelectronics in 1992, both from the University of Barcelona, Barcelona, Spain.

Since 1988, he has been with the Department of Electronics at the University of Barcelona, where he is currently Professor Titular in electronic engineering. His research interest is CMOS VLSI design.

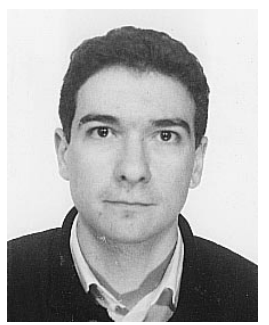

Carles Rubio received the degree in physics from the University of Barcelona, Barcelona, Spain, in 1992. He is currently pursuing the Ph.D. degree in microelectronics.

$\mathrm{He}$ is with the Electronic Materials \& Engineering Laboratory of the Electronics Department, University of Barcelona. Since 1993, he has been working on switched current and its applications in sigma-delta modulators for high-resolution analogto-digital conversions. He is currently a Professor of Microelectronics Design at the University of Barcelona. His research interests are in the areas of integrated circuit design and microsystems.

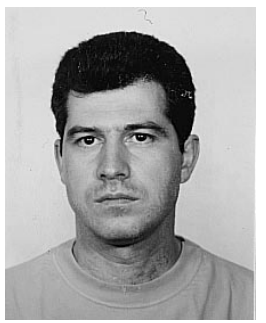

Stilianos K. Siskos (M'96) was born in 1956. He received the B.Sc. degree in physics from the Aristotle University of Thessaloniki, Thessaloniki, Greece, in 1980 and the M.Sc. and Ph.D. degrees in electronics from the University of Paul Sabatier de Toulouse, Toulouse, France, in 1983.

$\mathrm{He}$ has been a Lecturer at the School of Technology of Thessaloniki from 1985 to 1989 . He joined the Electronics Laboratory, Physics Department, Aristotle University of Thessaloniki, in 1989 as a Lecturer and, since 1993, he has been an Assistant Professor in the same laboratory. His current research interests are analog integrated circuit design, mixed built-in signal structures, current-mode integrated circuit design, sensor interfacing integrated circuits and design of signal processing circuits.

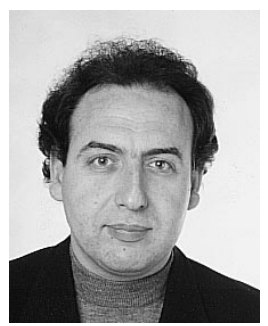

Theodore Laopoulos (M'89-SM'97) was born in Thessaloniki, Greece, in 1957. He received the B.Sc degree in physics and the Ph.D. degree in electronics from the Aristotle University of Thessaloniki, Thessaloniki, Greece.

$\mathrm{He}$ is currently Assistant Professor at the Electronics Laboratory, Physics Department, of the same university. His research interests are in the fields of circuits and systems for low-frequency instrumentation, measurement systems and techniques, sensor interfacing and control electronics, and currentmode analog integrated circuit design and applications. He is actively involved in research projects (National and European), collaboration projects with other European laboratories, has contributed more than 30 papers to scientific journals and conference proceedings, and has authored lecture notes in electronic circuits and applications.

Dr. Lapoulos is a member of the IEEE Imstrumentation and Measurement Society. He is currently serving as a Member of the Editorial Board of the new Instrumention and Measurement Magazine and as Associate Editor of the IEEE Transactions on Instrumentation and MEasurement 